\title{
TRIM59 is upregulated and promotes cell proliferation and migration in human osteosarcoma
}

\author{
JINQIAN LIANG $^{1}$, DAN XING ${ }^{2}$, ZHENG LI $^{1}$, JIANXIONG SHEN ${ }^{1}$, HONG ZHAO $^{1}$ and SHUGANG LI ${ }^{1}$ \\ ${ }^{1}$ Department of Orthopaedic Surgery, Peking Union Medical College Hospital, Beijing 100730; \\ ${ }^{2}$ Department of Orthopaedic Surgery, Tianjin Hospital, Tianjin 300211, P.R. China
}

Received April 20,2015; Accepted February 22, 2016

DOI: $10.3892 / \mathrm{mmr} .2016 .5183$

\begin{abstract}
Osteosarcoma is a prevalent type of cancer and has a high metastatic ability, particularly for metastasis to the lungs. Effective treatment strategies have improved, however, the detailed molecular mechanism underlying the onset of this malignancy remains to be fully elucidated. The current study investigated the role of the tripartite motif (TRIM) family protein TRIM59 in osteosarcoma growth and metastasis. It was identified that TRIM59 was overexpressed in clinical osteosarcoma tissues and cultured osteosarcoma cell lines. In addition, the MTT assay demonstrated that in U2OS and MG63 cells, knockdown of TRIM59 by specific siRNA inhibited proliferation, whereas overexpression of TRIM59 promoted cell proliferation. Furthermore, overexpression of TRIM59 significantly increased the U2OS cell migrative and invasive abilities in a Transwell chamber assay. In addition, TRIM59 was able to negatively regulate the protein levels of P53 without significantly affecting the mRNA levels in U2OS and MG63 cells. These data suggest the oncogenic abilities of TRIM59 in osteosarcoma, which promote osteosarcoma cell proliferation, migration and invasion.
\end{abstract}

\section{Introduction}

Osteosarcoma is one of the most commonly diagnosed malignant bone tumors in the clinic. It is the eighth leading type of childhood cancer and accounts for $\sim 2.4 \%$ of all malignancies in pediatric patients, and $20 \%$ of all primary bone cancers, thus is a significant health problem in this age group $(1,2)$. Osteosarcoma has a high tendency for metastasis, predominantly to the lungs (particularly the periphery of the lungs). One difficulty in the treatment of this malignancy is the resistance of osteosarcoma to conventional chemotherapy (3-5).

Correspondence to: Dr Hong Zhao, Department of Orthopaedic Surgery, Peking Union Medical College Hospital, 1 Shuaifuyuan, Wangfujing, Dongcheng, Beijing 100730, P.R. China E-mail: hongzhang1943@126.com

Key words: TRIM59, osteosarcoma, proliferation, migration, invasion, P53
Although the diagnosis and treatment of non-metastatic osteosarcoma has improved, patients with metastasis exhibit poor prognosis, with a 5-year event-free survival rate less than $20 \%(6-8)$. It is of high priority to elucidate novel methods for the diagnosis and treatment of early stage osteosarcoma.

The human tripartite motif (TRIM) family has greater than 77 members, of which the majority of the proteins belong to the E3 ubiquitin ligases, due to the highly conservative really interesting new gene (RING) domain. These proteins are involved in a variety of biological processes, including transcriptional regulation, membrane repair, cytoskeleton remodeling and oncogenesis. Certain members of this family, including TRIM13, TRIM19 and TRIM25 have been demonstrated to exert biological activity during human tumorigenesis in leukemia, breast and prostate cancer, through the regulation of transcriptional factors (9-13). These observations suggest that the TRIM proteins may serve vital roles in human tumorigenesis.

TRIM59, a surface molecule, has also been demonstrated to be involved in certain types of human cancer (14). It has been observed to be markedly increased in gastric cancer and prominently associated with the poor outcome of patients (14). The oncogenic features of TRIM59 were first characterized in 2011 (15). Furthermore, TRIM59 has been identified as a multiple tumor biomarker in human tumorigenesis (16). The biological activity of TRIM59 has been observed to be closely associated with the regulation of P53. TRIM59 interacts with P53, leading to P53 ubiquitination and degradation, and consequently promotes tumor growth and migration (14). In addition, TRIM59 was observed to exert its proto-oncogenic function through interaction with the Ras signaling pathway in a transgenic mouse model of prostate cancer (17). The exact role of TRIM59 in human osteosarcoma, however, remains to be fully elucidated.

P53 is a tumor suppressor gene that can be stimulated by cellular stress, such as oxidative stress. Once activated, P53 causes cell-cycle arrest, promotes DNA repair or induces apoptosis through different signaling pathways. Human P53 protein has three domains: The central DNA-binding domain, the N-terminal transcription-activation domain (TA) and the C-terminal oligomerization domain (18-20). Of these domains, the TA domain is important, due to the fact that it provides the structural basis for other molecules including murine double minute 2 (MDM2) and MDMX to bind to P53 and regulate P53 transcription $(21,22)$. P53 is highly inactivated in various 
tumor types (14) and numerous molecules have been reported to promote tumor progression via regulation of P53.

The current study aimed to investigate the role of TRIM59 in human osteosarcoma growth and metastasis. Specific small interfering RNA (siRNA) against TRIM59 and an expression plasmid for TRIM59 were used for the modulation of TRIM59. In addition, the expression levels of TRIM59 were determined in human osteosarcoma tissues and in cultured osteosarcoma cell lines. Furthermore, it was investigated whether TRIM59 had an effect on P53 expression, and the potential regulatory mechanisms were discussed.

\section{Materials and methods}

Human samples. Samples from 30 patients with osteosarcoma, who had been admitted to Tianjin Hospital (Tianjin, China) were collected. Matched adjacent non-cancerous tissues were surgically dissected at the same time as the cancerous tissues for each case and written consent was obtained from each patient. Furthermore, all experiments were conducted in compliance with the official polices and defined protocols.

Cell culture. The human osteosarcoma cell lines U2OS, MG63 and MNNG, and the non-cancerous cell line hFOB1.19, were obtained from the Type Culture Collection of the Chinese Academy of Sciences (Shanghai, China). The three cancer cell lines were maintained in Eagle's minimum essential medium (EMEM) media (Gibco; Thermo Fisher Scientific, Inc., Waltham, MA, USA) supplemented with $10 \%$ fetal bovine serum (FBS; Gibco; Thermo Fisher Scientific, Inc.) and hFOB1.19 was cultured in Dulbecco's modified Eagle's medium supplemented with $10 \%$ FBS.

Total RNA extraction and cDNA synthesis. The total RNA from human tissues and cultured cells were extracted with TRIzol Reagent (Takara Bio, Inc., Otsu, Japan), according to the manufacturer's instructions. The quality and concentration of the isolated RNAs were determined by measuring absorbance with Nanodrop 2000 (Thermo Fisher Scientific, Inc.). First-strand cDNA (10 ng) was reverse transcribed using the PrimeScript RT Master Mix Perfect Real Time (Takara Bio, Inc.).

Reverse transcription-quantitative polymerase chain reaction (RT-qPCR). All RT-qPCR reactions were performed using an ABI PRISM 7900 Real-Time System with the SYBR Premix Ex Taq kit (Takara Bio, Inc.). The cycling protocol was as follows: Initial denaturation step at $95^{\circ} \mathrm{C}$ for $2 \mathrm{~min}, 35$ cycles of the three-step cycling program consisting of $30 \mathrm{sec}$ at $95^{\circ} \mathrm{C}$ (denaturation), $1 \mathrm{~min}$ at $55^{\circ} \mathrm{C}$ (primer annealing) and $30 \mathrm{sec}$ at $72^{\circ} \mathrm{C}$ (elongation), then a final extension step for $10 \mathrm{~min}$ at $72^{\circ} \mathrm{C}$. Amplification products were determined by $1 \%(\mathrm{w} / \mathrm{v})$ agarose gels (Bio-Rad Laboratories, Inc., Hercules, CA, USA). The primers used are presented in Table I, and the internal reference gene glyceraldehyde-3-phosphate dehydrogenase (GAPDH) was used as the internal control. All quantitative data were normalized to the internal control gene.

Western blot analysis. Total proteins from clinical tissues and cultured cells were extracted. For human tissues, each sample was cut into pieces and incubated in lysis buffer (Beyotime
Institute of Biotechnology, Haimen, China) with phenylmethanesulfonyl fluoride protease inhibitor (Sigma-Aldrich, St. Louis, MO, USA) for $40 \mathrm{~min}$ at $4^{\circ} \mathrm{C}$. Subsequent to ultra-centrifugation at $12,000 \mathrm{x} \mathrm{g}$ at $4^{\circ} \mathrm{C}$ for $1 \mathrm{~h}$, the supernatant was used for immunoblot analysis. For cultured cells, when confluence reached $95 \%$, cells were washed twice with phosphate-buffered saline (Gibco; Thermo Fisher Scientific, Inc.) followed by lysis with the lysis buffer ( $\mathrm{pH} 7.5)$ to generate the whole protein lysate. Equal amounts of protein $(50 \mu \mathrm{g})$ were loaded onto each lane using a 12\% sodium dodecyl sulfate-polyacrylimide gel electrophoresis gel. GAPDH was synchronously developed as a loading control. The membranes were blocked in 5\% skimmed milk for $1 \mathrm{~h}$ at room temperature, and incubated with rabbit polyclonal TRIM59 (1:1,000; cat. no. sc-134123), P53 (1:1,000; cat. no. sc-6243) and GAPDH (1:1,000; cat. no. sc-25778; all purchased from Santa Cruz Biotechnology, Inc., Dallas, TX, USA) at $4^{\circ} \mathrm{C}$ overnight. Subsequent to primary incubation, the membranes were further incubated with a goat anti-rabbit IgG (1:200; Santa Cruz Biotechnology, Inc.; cat. no. sc-45101) at $37^{\circ} \mathrm{C}$ for $1 \mathrm{~h}$. Immunoreactivity was determined with enhanced chemiluminescent autoradiography (Thermo Fisher Scientific, Inc.) and each experiment was repeated at least three times.

siRNA interference and plasmid transfection. For knockdown of TRIM59, a specific siRNA was designed and chemically synthesized by Santa Cruz Biotechnology, Inc. The pcDNA3.1-TRIM59 expression plasmid was constructed according to routine protocols. In brief, primers for cloning and amplifying human TRIM59 gene were designed. The forward primer sequence was 5'-AATGCACAATTTTGAGGAAG-3' and the reverse primer sequence was 5'-TCCACACAAATT CCTTCAAC-3'. The transfection assay was performed with Lipofectamine 2000 transfection reagent (Life Technologies; Thermo Fisher Scientific, Inc.) according to the manufacturer's instructions. Subsequent to $6 \mathrm{~h}$ of transfection, the media was replaced with fresh EMEM media with $10 \%$ FBS.

Cell proliferation assay. U2OS and MG63 cells were seeded into 96-well plates (3,000 cells/well) and allowed to grow overnight. Cells were then transfected with $10 \mu \mathrm{M}$ siRNA or TRIM59 plasmid, followed by incubation in EMEM media for an additional $72 \mathrm{~h}$ at $37^{\circ} \mathrm{C}$. Cell viability was determined for 5 consecutive days with 3-(4,5-dimethylthiazol-2-yl)-2,5-dipheny ltetrazoliumbromide (MTT; Beyotime Institute of Biotechnology) solution. For each monitored day, $2 \mathrm{mg} / \mathrm{ml}$ MTT solution was added to each well. Subsequent to incubation for $4 \mathrm{~h}$ at $37^{\circ} \mathrm{C}$, media was discarded and $200 \mu \mathrm{l}$ dimethyl sulfoxide (OriGene Technologies, Inc., Rockville, MD, USA) was added into each well. The plate was shaken for $5 \mathrm{~min}$ and the optical density was measured at a wavelength of $570 \mathrm{~nm}$ using the SpectraMax M5/M5e microplate reader (Molecular Devices LLC, Sunnyvale, CA, USA).

Transwell assay. The U2OS cell line was cultured in 24-well plates and transfected with specific TRIM59 siRNA or plasmids. Cells were harvested $48 \mathrm{~h}$ post-transfection in serum-free EMEM as a single cell suspension. A total of $150 \mu 1$ cell suspension was seeded into the upper chamber (Corning Incorporated, Corning, NY, USA), while the lower chamber was filled with 
Table I. Primers used in reverse transcription-quantitative polymerase chain reaction.

\begin{tabular}{lcl}
\hline Gene & Gene ID & \multicolumn{1}{c}{ Primer nucleotide sequences } \\
\hline Tripartite motif family protein 59 & 286827 & $\begin{array}{l}\text { Forward: 5'-TACGAGAGCAGCAGCTTGAA-3' } \\
\text { Reverse: 5'-ACGGGTTGAACCTCAGGAAG-3' }\end{array}$ \\
P53 & 7157 & $\begin{array}{l}\text { Forward: 5'-GAGGTTGGCTCTGACTGTACC-3' } \\
\text { Reverse: 5'-TCCGTCCCAGTAGATTACCAC-3' }\end{array}$ \\
Glyceraldehyde 3-phosphate dehydrogenase & 2597 & $\begin{array}{l}\text { Forward: 5'-GTGGACATCCGCAAAGAC-3' } \\
\text { Reverse: 5'-AAAGGGTGTAACGCAACTA-3' }\end{array}$ \\
\hline
\end{tabular}

$600 \mu 1$ EMEM supplemented with 10\% FBS. For the invasion assay, the chamber was coated with Matrigel (Corning, Inc., Corning, NY, USA) $6 \mathrm{~h}$ prior to seeding cells into the chamber. Subsequent to incubation at $37^{\circ} \mathrm{C}$ for $12 \mathrm{~h}$, cells were fixed with ice-cold methanol for $20 \mathrm{~min}$ and stained with $0.1 \%$ crystal violet (Beyotime Institute of Biotechnology) for $5 \mathrm{~min}$. The images were obtained under a Nikon XP-330C microscope at a magnification of x200 (Nikon Corporation, Tokyo, Japan). This assay was repeated a minimum of three times, each repeat in duplicate.

Statistical analysis. The results were presented as the mean \pm standard deviation. Statistical analysis was conducted with Student's t-test using the SPSS software, version 18.0 (SPSS, Inc, Chicago, IL, USA). P $<0.05$ was considered to indicate a statistically significant difference. All experiments were repeated a minimum of three times unless otherwise stated.

\section{Results}

TRIM59 is overexpressed in human osteosarcoma tissues and cultured osteosarcoma cells. To clarify the role of TRIM59 in osteosarcoma, the expression of TRIM59 was initially examined in 30 cases of clinical human osteosarcoma tissues and their adjacent normal tissues. Western blot analysis indicating that the protein level of TRIM59 was significantly increased in 28 cases of osteosarcoma tissues compared with the adjacent normal tissues. The remaining 2 cases were not observed to exhibit significantly different expression levels of TRIM59 between cancerous and non-cancerous tissues. The western blots of 5 representative cases that exhibited higher protein levels of TRIM59 are presented in Fig. 1A. The RT-qPCR assay also indicated that the average mRNA levels of TRIM59 in the clinical osteosarcoma tissues were approximately three-fold of that of the adjacent tissues (n=30; Fig. 1B). U2OS, MG63 and MNNG cells are three osteosarcoma cell lines that have been used in previous studies, while the hFOB1.19 cell line is a commercial human osteoblast cell line, which is steadily-transfected with SV40. Fig. 1C indicated that the protein levels of TRIM59 in the three osteosarcoma cell lines were markedly higher than that of the control non-osteosarcoma cell line hFOB1.19. Among the osteosarcoma cell lines, U2OS and MG163 cells exhibited the highest protein levels of TRIM59, making them the optimal cell lines for further investigation. These data indicated that TRIM59 is highly expressed in human osteosarcoma tissues and in cultured cell lines.
Transfection efficiency of the specific siRNA against TRIM59 (siTRIM59) and the TRIM59 expression plasmid. To further examine the role of TRIM59 in osteosarcoma progression, two osteosarcoma cell lines (U2OS and MG63) were transfected with specific siRNA against TRIM59 (siTRIM59) and the TRIM59 plasmid. RT-qPCR data demonstrated that the relative mRNA levels of TRIM59 in siTRIM59-transfected U2OS cells were reduced by $\sim 60 \%$ compared with control cells; while the levels were reduced by $\sim 70 \%$ in siTRIM59-transfected MG63 cells (Fig. 2A). On the contrary, the mRNA level of TRIM59 was increased by 3.2-fold in U2OS cells and 3.7-fold in MG63 cells, when the pcDNA3.1-TRIM59 plasmid was transfected (Fig. 2B). The results of western blot analysis were consistent, indicating that the protein levels of TRIM59 were notably suppressed by transfection of siTRIM59, while markedly increased by transfection of the TRIM59 plasmid in the two cell lines (Fig. 2C). These results confirmed that transfection was effective, and confirmed the efficiency of specific siTRIM59 and the TRIM59 plasmid in the modulation of TRIM59 expression.

TRIM59 increases the cell proliferation ability in the U2OS and MG63 osteosarcoma cell lines. TRIM59 has been reported to function as an activator of cell proliferation in gastric cancer (14). Thus, the effects of TRIM59 modulation on osteosarcoma cell proliferation were investigated. The MTT assay indicated that cell proliferative rates were not significantly different in the first three days among three groups, only a marginal difference was observed. However, on the fourth and fifth days, the proliferative rate of siTRIM59-transfected U2OS cells was significantly reduced, by $12.5 \%$ and $23.3 \%$, respectively, compared with the control U2OS cells (Fig. 3A). Similar results were observed in the MG63 cells, with significant reductions in the proliferative rate subsequent to transfection of siTRIM59 (Fig. 3B). By contrast, overexpression of TRIM59 increased the proliferative rate by $30 \%$ in U2OS cells and $27 \%$ in MG63 cells on the fourth day. Cell proliferation was further increased on the fifth day, by $31 \%$ and $31.2 \%$ in U2OS and MG63 cells, respectively (Fig. 3C and D). These data suggest that TRIM59 may promote cell proliferation in osteosarcoma.

TRIM59 promotes cell migration and invasion in osteosarcoma. Osteosarcoma has a high tendency to metastasize; consequently it was investigated whether TRIM59 served a role in this process. The Transwell assay, together with the subsequent crystal violet staining, indicated that the number of migrated cells 

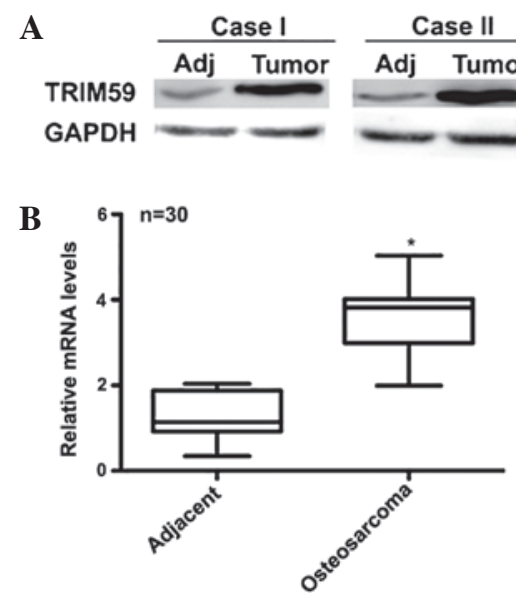

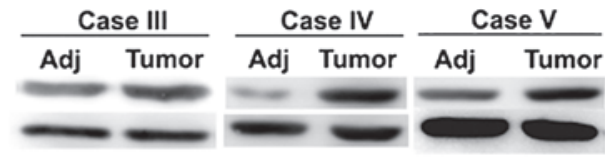

C

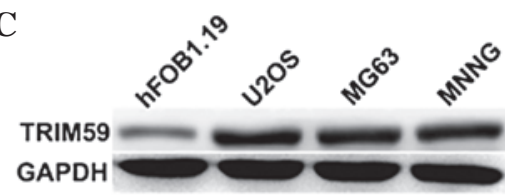

Figure 1. TRIM59 is overexpressed in human osteosarcoma tissues and cultured cells. (A) Representative western blot bands, indicative of the overexpression of TRIM59 (vs. the adjacent non-cancerous tissues) observed in the majority of osteosarcoma tissues (28/30). (B) Reverse transcription-quantitative polymerase chain reaction analysis of 30 clinical osteosarcoma tissues and their adjacent tissues demonstrated a significant difference between the osteosarcoma and adjacent tissues. ${ }^{*} \mathrm{P}<0.05$ vs. adjacent tissues. (C) In all osteosarcoma cell lines, TRIM59 was overexpressed compared with the non-osteosarcoma cell line. Data presented are representative of three independent experiments. TRIM59, tripartite motif family protein 59; GAPDH, glyceraldehyde 3-phosphate dehydrogenase.

A

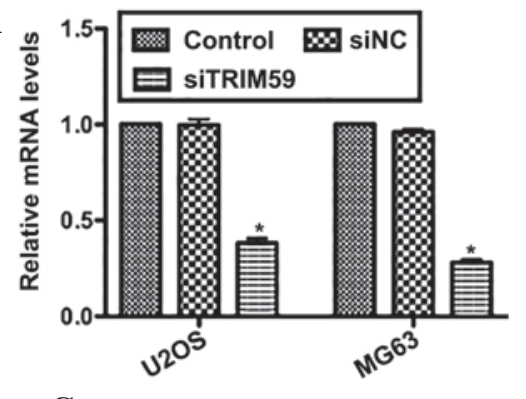

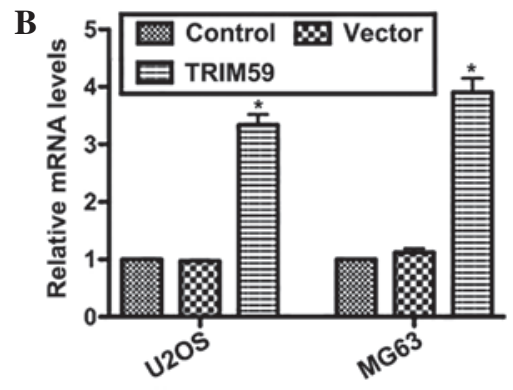

C

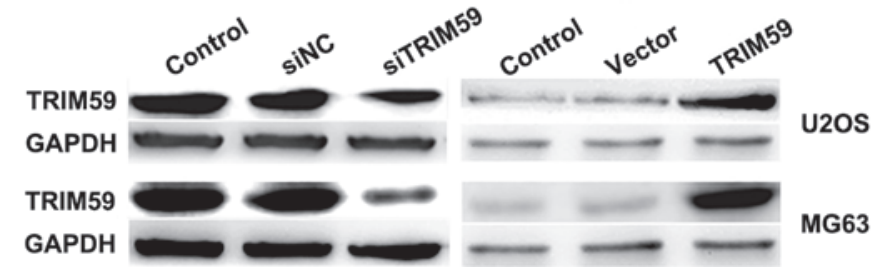

Figure 2. Transfection efficiency of siTRIM59 and TRIM59 plasmids. (A) Relative mRNA levels of TRIM59 when siTRIM59 was transfected in U2OS and MG63 cell lines. (B) Relative mRNA levels of TRIM59 when the TRIM59 plasmid was transfected into the U2OS and MG63 cell lines. siNC indicates non-specific siRNA, and vector indicates pcDNA3.1. "P<0.05 vs. control. (C) The protein levels of TRIM59 when transfected with siTRIM59 and TRIM59 plasmids in the U2OS and MG63 osteosarcoma cell lines. GAPDH was included as the internal control. si, small interfering; TRIM59, tripartite motif family protein 59; GAPDH, glyceraldehyde 3-phosphate dehydrogenase.

were markedly different between the experimental and control groups (Fig. 4A). Counting of the migrated cells indicated that compared with the corresponding control group, the number of migrated cells attached to the permeable membrane was significantly reduced, by $\sim 50 \%$ in the siTRIM59-transfected group, however was significantly increased by $30 \%$ in TRIM59-treated U2OS cells (Fig. 4B and C). For the invasion assay, U2OS cells with TRIM59 knockdown exhibited lower abilities, while cells with TRIM59 overexpression exhibited higher abilities to invade through the Matrigel and migrate through the pores (Fig. 4B and C). These data support the hypothesis that TRIM59 contributes to cell migration and invasion in osteosarcoma.

TRIM59 inhibits P53 expression at the protein level. The tumor suppressor gene P53 works as a "guardian" of the human genome and mutations of P53 have been reported to trigger human carcinogenesis (23). Therefore, it was investigated whether TRIM59 had any effects on P53 expression in osteosarcoma. The RT-qPCR assay indicated that the mRNA levels of P53 were not significantly altered in response to knockdown or overexpression of TRIM59 in the two osteosarcoma cell lines (Fig. 5A and B). However, protein levels of P53 were markedly altered by modulation of TRIM59. Knockdown of TRIM59 increased, whereas overexpression of TRIM59 reduced, the expression of P53 protein in U2OS (Fig. 5C) and MG63 (Fig. 5D) cells. These data suggest that TRIM59 may regulate P53 expression in osteosarcoma, however only at a protein level.

\section{Discussion}

Osteosarcoma is the fifth most frequent malignancy among adolescents between 15 and 19 years old, particularly in boys 

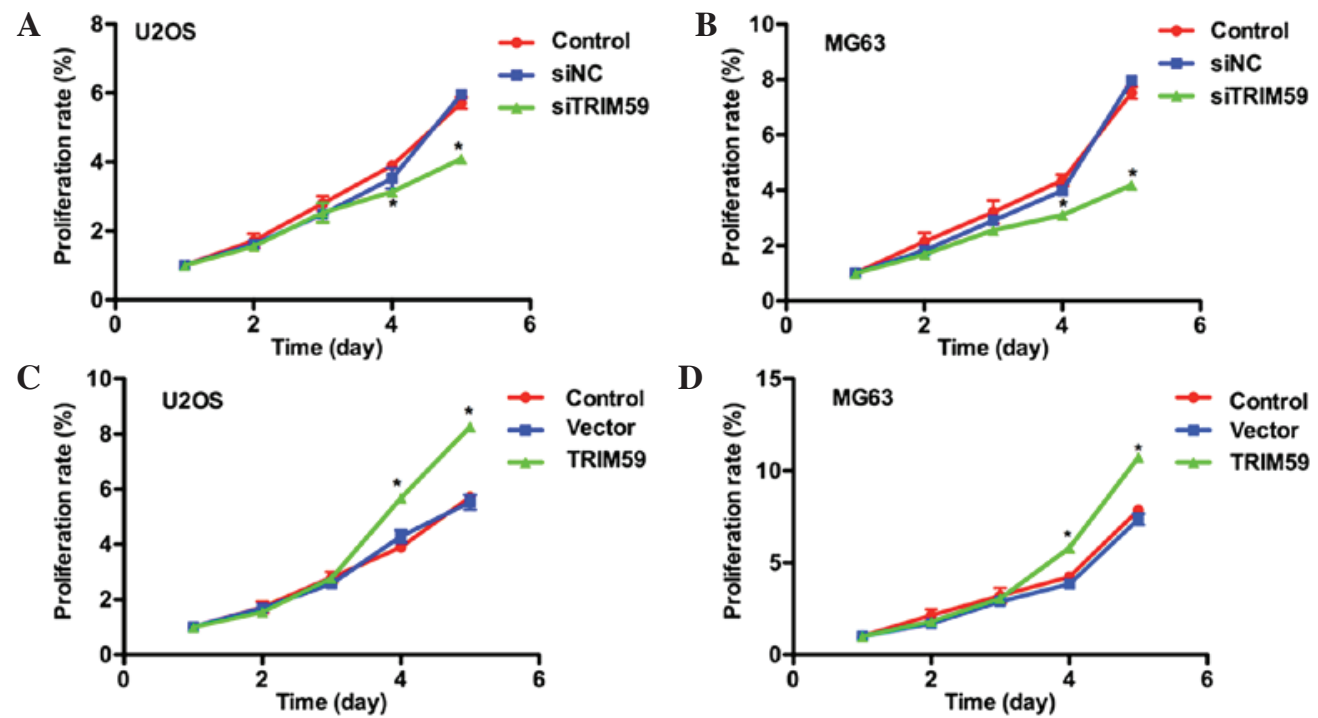

Figure 3. TRIM59 inhibits cell proliferation in the two osteosarcoma cell lines. (A) The proliferative rate was reduced by $12.5 \%$ on day 4 and $23.3 \%$ on day 5 when siTRIM59 was transfected into U2OS cells. (B) The proliferative rate was reduced by $31.5 \%$ on day 4 and $50 \%$ on day 5 when the siTRIM59 was transfected into MG63 cells. (C) The proliferative rate was increased by $30 \%$ on day 4 and $31 \%$ on day 5 when the TRIM59 plasmid was transfected into U2OS cells. (D) The proliferative rate was increased by $27 \%$ on day 4 and $31.2 \%$ on day 5 when the TRIM59 plasmid was transfected into MG63 cells. "P<0.05 vs. control. TRIM59, tripartite motif family protein 59; si, small interfering.

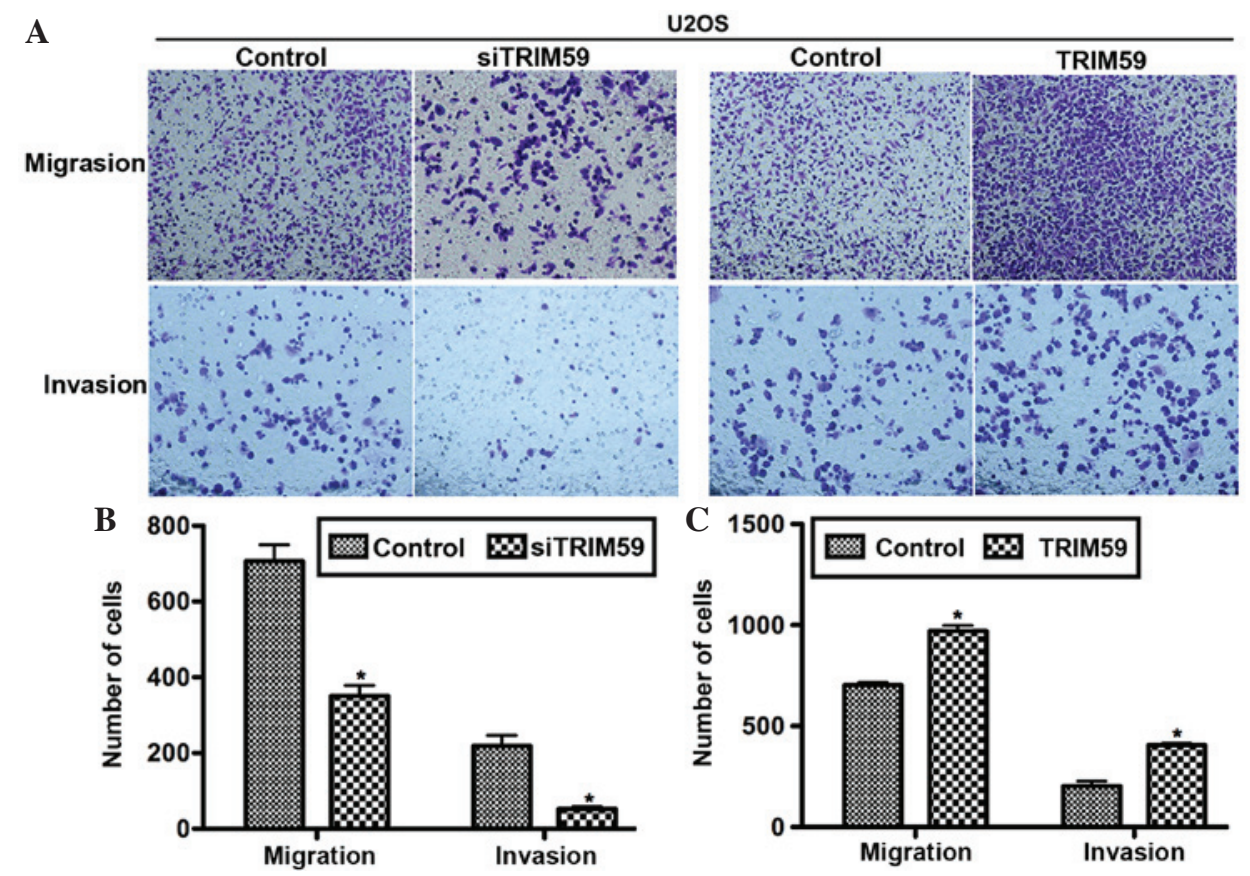

Figure 4. TRIM59 promotes cell migration and invasion in osteosarcoma. (A) U2OS cell Transwell assay. Cells treated with siTRIM59 exhibited a reduced ability for migration and invasion, whereas TRIM59-transfected cells exhibited an increased ability. Quantification of the Transwell assay with (B) siTRIM59 and (C) TRIM59 transfection. Cells were allowed to migrate or invade for $12 \mathrm{~h}$ subsequent to plating. "P<0.05 vs. control. TRIM59, tripartite motif family protein 59; si, small interfering.

and African-American children (24,25). Familial cases, bone dysplasias, Li-Fraumeni syndrome and Rothmund-Thomson syndrome are the main manifestations of osteosarcoma observed clinically (26). In the USA, it was reported that one third of patients suffering from osteosarcoma die each year, with the majority of these victims being teenagers (27). As a result, further research is required to aid in the discovery of novel diagnostic and prognostic biomarkers, and an improved understanding of the mechanisms for osteosarcoma development.
In the present study, it was demonstrated that TRIM59 was overexpressed in clinical osteosarcoma tissues at mRNA and protein levels. By employing RNAi and plasmid transfection techniques, it was indicated that knockdown of TRIM59 restricted, whereas overexpression of TRIM59 promoted, the cell proliferative, migratory and invasive abilities of osteosarcoma U2OS and MG63 cells. Thus it is suggested that TRIM59 is a key mediator of osteosarcoma progression. However, in vivo data, such as a mouse model of osteosarcoma, 

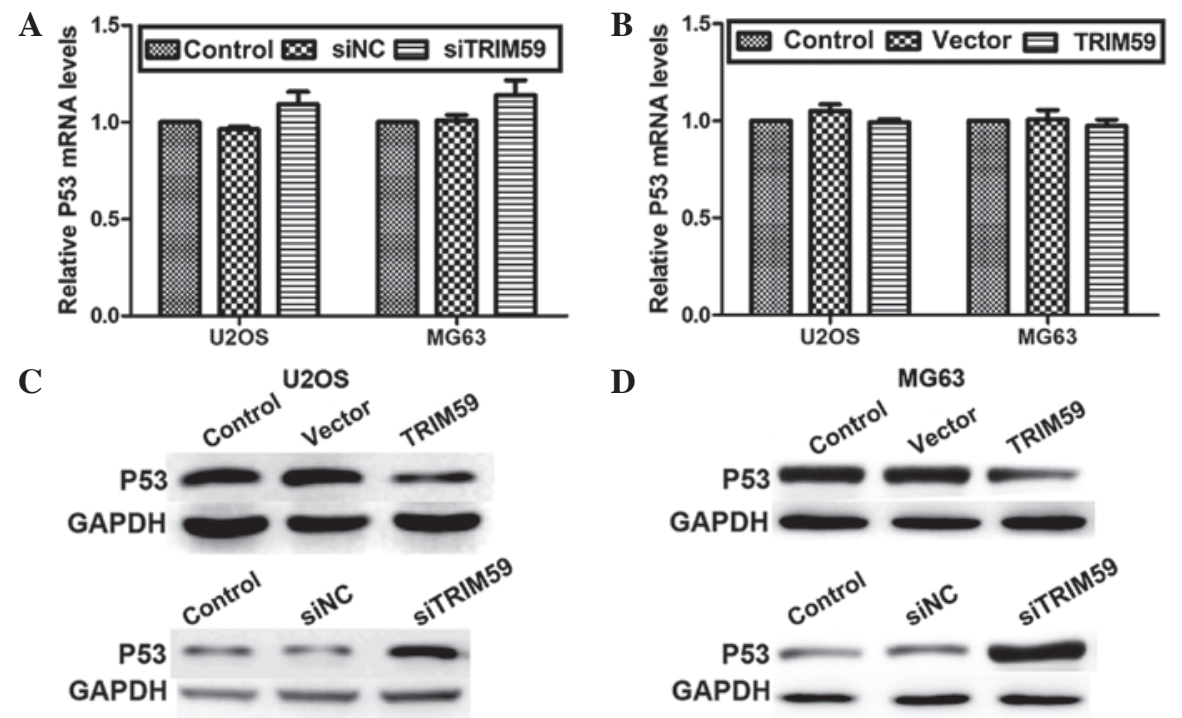

Figure 5. TRIM59 inhibited P53 expression at a protein level. (A) Knockdown of TRIM59 in U2OS and MG63 cells did not alter the P53 mRNA level. (B) Overexpression of TRIM59 in U2OS and MG63 cells did not alter the P53 mRNA level. In (C) U2OS and (D) MG63 osteosarcoma cells, P53 protein levels were reduced when the TRIM59 plasmid was transfected, and were increased subsequent to the transfection of siTRIM59. TRIM59, tripartite motif family protein 59; si, small interfering.
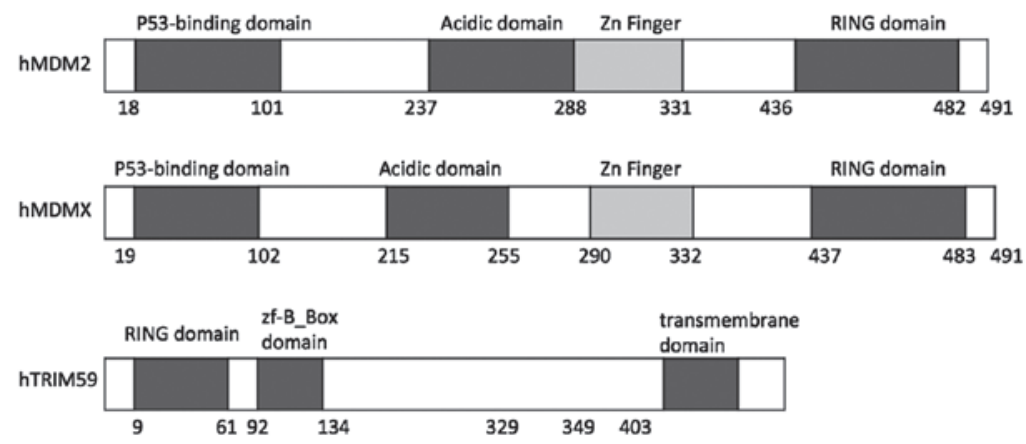

Figure 6. The schematic structure of human MDM2, MDMX and TRIM59. MDM2 and MDMX have four domains: The P53-binding, acidic, Zn finger and RING domains. TRIM59 has three domains: The RING, zf-B_Box and transmembrane domains. MDM2, murine double minute 2; TRIM59, tripartite motif family protein 59. RING, really interesting new gene.

is required in order to further confirm the critical role of TRIM59 in osteosarcoma progression.

Notably, it was identified that knockdown of TRIM59 in the two cell lines increased the protein levels of P53, without significantly affecting the mRNA levels. TRIM59 belongs to the E3 ubiquitin ligase family, which promotes ubiquitin-mediated degradation of target proteins (28). The regulation of P53 by TRIM59 in osteosarcoma cells would suggest that TRIM59 mediated osteosarcoma progression, potentially via regulation of P53 (predominantly by ubiquitin-mediated degradation). It has also been previously reported that TRIM59 is upregulated in gastric cancer and promotes the ubiquitination and degradation of P53 (14). This indicates that ubiquitin ligase is important in the activity of TRIM59. How TRIM59 regulates the ubiquitination and degradation of P53 remains unclear. P53 inhibits tumor development through various approaches including induction of apoptosis and autophagy $(26,29)$. MDM2 and its homolog MDMX (also termed MDM4) are the key suppressors of P53 in multiple tumor types $(30,31)$. As presented in Fig. 6, the MDM2 N-terminal fragment (18-101 amino acids)/MDMX
$\mathrm{N}$-terminal fragment (19-102 amino acids) has the ability to bind with the P53 transactivation domain peptide $(21,32)$. In addition, the RING domain of MDMX heterodimerizes with that of MDM2, leading to the recruitment of E2 ubiquitin-conjugating enzyme and the final ubiquitination and degradation of P53 (32-34). One possible mechanism for TRIM59 regulation of P53 is TRIM59-mediated promotion of P53 degradation through its RING domain, as observed in MDMX and MDM2. The RING domains of MDM2 and MDMX are important in their binding with P53, and TRIM59 also has a RING domain at its N-terminal (Fig. 6). This structural similarity between TRIM59 and MDM2/MDMX additionally suggests a similar regulatory mechanism for P53. However, the specific function of the RING domain in TRIM59 remains to be fully elucidated, thus further investigation is required.

In conclusion, TRIM59 was observed to be upregulated in clinical osteosarcoma tissues and the cultured osteosarcoma cell lines U2OS and MG63. Knockdown of TRIM59 with specific siRNA inhibited the cell proliferative, migratory and invasive abilities of osteosarcoma cells. By contrast, when 
TRIM59 was overexpressed in osteosarcoma cells, increased cell proliferative rates and migratory abilities were observed. The regulation of P53 by TRIM59 at its protein level may suggest that TRIM59 promotes osteosarcoma progression by ubiquitination and degradation of P53.

\section{Acknowledgements}

The present study was sponsored by the Natural Science Foundation of China (grant no. 81301596).

\section{References}

1. Messerschmitt PJ, Garcia RM, Abdul-Karim FW, Greenfield EM and Getty PJ: Osteosarcoma. J Am Acad Orthop Surg 17: 515-527, 2009.

2. Mirabello L, Troisi RJ and Savage SA: Osteosarcoma incidence and survival rates from 1973 to 2004: Data from the surveillance, epidemiology, and end results program. Cancer 115: 1531-1543, 2009.

3. Bacci G, Rocca M, Salone M, Balladelli A, Ferrari S, Palmerini E, Forni C and Briccoli A: High grade osteosarcoma of the extremities with lung metastases at presentation: Treatment with neoadjuvant chemotherapy and simultaneous resection of primary and metastatic lesions. J Surg Oncol 98: 415-420, 2008.

4. Bielack SS, Carrle D, Hardes J, Schuck A and Paulussen M Bone tumors in adolescents and young adults. Curr Treat Options Oncol 9: 67-80, 2008.

5. Hughes DP: Strategies for the targeted delivery of therapeutics for osteosarcoma. Expert Opin Drug Deliv 6: 1311-1321, 2009.

6. Ferrari S, Smeland S, Mercuri M, Bertoni F, Longhi A, Ruggieri P, Alvegard TA, Picci P, Capanna R, Bernini G, et al: Neoadjuvant chemotherapy with high-dose ifosfamide, high-dose methotrexate, cisplatin, and doxorubicin for patients with localized osteosarcoma of the extremity: A joint study by the Italian and Scandinavian sarcoma groups. J Clin Oncol 23: 8845-8852, 2005.

7. Mialou V, Philip T, Kalifa C, Perol D, Gentet JC, Marec-Berard P, Pacquement H, Chastagner P, Defaschelles AS and Hartmann O: Metastatic osteosarcoma at diagnosis: Prognostic factors and long-term outcome-the French pediatric experience. Cancer 104: 1100-1109, 2005.

8. Smeland S, Müller C, Alvegard TA, Wiklund T, Wiebe T, Björk O, Stenwig AE, Willén H, Holmström T, Follerås G, et al: Scandinavian sarcoma group osteosarcoma study SSG VIII: Prognostic factors for outcome and the role of replacement salvage chemotherapy for poor histological responders. Eur J Cancer 39: 488-494, 2003.

9. Klugbauer S and Rabes HM: The transcription coactivator HTIF1 and a related protein are fused to the RET receptor tyrosine kinase in childhood papillary thyroid carcinomas. Oncogene 18: 4388-4393, 1999.

10. Hatakeyama S: TRIM proteins and cancer. Nat Rev Cancer 11: 792-804, 2011.

11. de Thé H, Lavau C, Marchio A, Chomienne C, Degos L and Dejean A: The PML-RAR alpha fusion mRNA generated by the $t(15 ; 17)$ translocation in acute promyelocytic leukemia encodes a functionally altered RAR. Cell 66: 675-684, 1991.

12. Cambiaghi V, Giuliani V, Lombardi S, Marinelli C, Toffalorio F and Pelicci PG: TRIM proteins in cancer. Adv Exp Med Biol 770: 77-91, 2012.

13. Le Douarin B, Zechel C, Garnier JM, Lutz Y, Tora L, Pierrat B, Heery D, Gronemeyer H, Chambon P and Losson R: The N-terminal part of TIF1, a putative mediator of the ligand-dependent activation function (AF-2) of nuclear receptors, is fused to B-raf in the oncogenic protein T18. EMBO J 14: 2020-2033, 1995.

14. Zhou Z, Ji Z, Wang Y, Li J, Cao H, Zhu HH and Gao WQ: TRIM59 Is up-regulated in gastric tumors, promoting ubiquitination and degradation of p53. Gastroenterology 147: 1043-1054, 2014.

15. Valiyeva F, Jiang F, Elmaadawi A, Moussa M, Yee SP, Raptis L, Izawa JI, Yang BB, Greenberg NM, Wang F and Xuan JW: Characterization of the oncogenic activity of the novel TRIM59 gene in mouse cancer models. Mol Cancer Ther 10: 1229-1240, 2011.
16. Khatamianfar V, Valiyeva F, Rennie PS, Lu WY, Yang BB, Bauman GS, Moussa M and Xuan JW: TRIM59, a novel multiple cancer biomarker for immunohistochemical detection of tumorigenesis. BMJ Open 2: pii.e001410, 2012.

17. Valiyeva F, Jiang F, Elmaadawi A, Moussa M, Yee SP, Raptis L, Izawa JI, Yang BB, Greenberg NM, Wang F and Xuan JW: Characterization of the oncogenic activity of the novel TRIM59 gene in mouse cancer models. Mol Cancer Ther 10: 1229-1240, 2011.

18. Mavinahalli JN, Madhumalar A, Beuerman RW, Lane DP and Verma C: Differences in the transactivation domains of p53 family members: A computational study. BMC Genomics 11 (Suppl 1): S5, 2010.

19. Muller PA and Vousden KH: Mutant p53 in cancer: New functions and therapeutic opportunities. Cancer Cell 25: 304-317, 2014.

20. Sauer M, Bretz AC, Beinoraviciute-Kellner R, Beitzinger M, Burek C, Rosenwald A, Harms GS and Stiewe T: C-terminal diversity within the p53 family accounts for differences in DNA binding and transcriptional activity. Nucleic Acids Res 36: 1900-1912, 2008.

21. Kussie PH, Gorina S, Marechal V, Elenbaas B, Moreau J, Levine AJ and Pavletich NP: Structure of the MDM2 oncoprotein bound to the p53 tumor suppressor transactivation domain. Science 274: 948-953, 1996.

22. Momand J, Zambetti GP, Olson DC, George D and Levine AJ: The mdm-2 oncogene product forms a complex with the p53 protein and inhibits p53-mediated transactivation. Cell 69: 1237-1245, 1992.

23. Overholtzer M, Rao PH, Favis R, Lu XY, Elowitz MB, Barany F, Ladanyi M, Gorlick R and Levine AJ: The presence of p53 mutations in human osteosarcomas correlates with high levels of genomic instability. Proc Natl Acad Sci USA 100: 11547-11552, 2003.

24. Marina N, Gebhardt M, Teot L and Gorlick R: Biology and therapeutic advances for pediatric osteosarcoma. Oncologist 9: 422-441, 2004.

25. Sandberg AA and Bridge JA: Updates on the cytogenetics and molecular genetics of bone and soft tissue tumors: Osteosarcoma and related tumors. Cancer Genet Cytogenet 145: 1-30, 2003.

26. Ottaviani $\mathrm{G}$ and Jaffe N: The etiology of osteosarcoma. Cancer Treat Res 152: 15-32, 2009.

27. Kim FM, Hayes C, Williams PL, Whitford GM, Joshipura KJ, Hoover RN and Douglass CW; National Osteosarcoma Etiology Group: An assessment of bone fluoride and osteosarcoma. J Dent Res 90: 1171-1176, 2011.

28. Kondo T, Watanabe $M$ and Hatakeyama S: TRIM59 interacts with ECSIT and negatively regulates NF-kB and IRF-3/7-mediated signal pathways. Biochem Biophys Res Commun 422: 501-507, 2012.

29. Vousden KH and Prives C: Blinded by the light: The growing complexity of p53. Cell 137: 413-431, 2009.

30. Pei D, Zhang Y and Zheng J: Regulation of p53: A collaboration between Mdm2 and Mdmx. Oncotarget 3: 228-235, 2012.

31. Wang $X$ and Jiang $X: M d m 2$ and $M d m X$ partner to regulate p53. FEBS Lett 586: 1390-1396, 2012.

32. Shvarts A, Steegenga WT, Riteco N, van Laar T, Dekker P, Bazuine M, van Ham RC, van der Houven van Oordt W, Hateboer G, van der Eb AJ and Jochemsen AG: MDMX: A novel p53-binding protein with some functional properties of MDM2. EMBO J 15: 5349-5357, 1996.

33. Danovi D, Meulmeester E, Pasini D, Migliorini D, Capra M, Frenk R, de Graaf P, Francoz S, Gasparini P, Gobbi A, et al: Amplification of Mdmx (or Mdm4) directly contributes to tumor formation by inhibiting p53 tumor suppressor activity. Mol Cell Biol 24: 5835-5843, 2004.

34. Zdzalik M, Pustelny K, Kedracka-Krok S, Huben K, Pecak A, Wladyka B, Jankowski S, Dubin A, Potempa J and Dubin G: Interaction of regulators $\mathrm{Mdm} 2$ and $\mathrm{Mdmx}$ with transcription factors p53, p63 and p73. Cell Cycle 9: 4584-4591, 2010 . 